\title{
Bienestar subjetivo: el papel de la rumia, optimismo, resiliencia y capacidad de recibir apoyo
}

\author{
Individual well-being: the role of rumination, optimism, resilience and ability to \\ receive support
}

\section{Bem-estar subjetivo: papel da ruminação, otimismo, resiliência e capacidade de receber apoio}

\author{
Rozzana Sánchez-Aragón ${ }^{\text {I }}$, ORCID 0000-0002-5952-8972 \\ ${ }^{1}$ Universidad Nacional Autónoma de México (UNAM). México
}

\begin{abstract}
Resumen: El bienestar subjetivo comprende experiencias emocionales positivas/negativas, prosperidad y satisfacción con la vida. Además, depende de múltiples factores psicosociales como: la rumia, el optimismo, la resiliencia y la capacidad para recibir apoyo, mismos que han sido estudiados en particular y necesitan ser examinados en conjunto. Por ello, este estudio se propuso: 1) identificar el efecto de las variables mencionadas en el bienestar subjetivo en adultos, y 2) explorar sus diferencias a partir del sexo, edad y escolaridad. Se contó con la participación voluntaria de 404 adultos mexicanos de entre 18 y 64 años $(M=37.56)$, con escolaridad mínima de secundaria. Los resultados muestran el papel significativo de algunos factores del optimismo, resiliencia y rumia en la predicción del bienestar subjetivo, así como diferencias en auto-confianza (optimismo), experiencia emocional negativa (bienestar subjetivo) y capacidad de recibir apoyo entre sexos, y la tendencia a experimentar más optimismo, resiliencia y bienestar conforme se tiene más edad y escolaridad. Estos resultados muestran como los atributos positivos y la experiencia de vida se asociacian con otras experiencias positivas en pro del bienestar del individuo.
\end{abstract}

Palabras clave: bienestar; rumia; optimismo; resiliencia; apoyo

Abstract: Subjective well-being includes having positive / negative emotional experiences, prosperity and satisfaction with life. In addition, it depends on multiple psychosocial factors such as: rumination, optimism, resilience and the ability to receive support, which have been studied in particular and need to be examined together. Therefore, this study was set out to: 1) Identify the effect of the aforementioned variables on subjective well-being in adults, and 2) Explore their differences based on gender, age and schooling. There was a voluntary participation of 404 Mexican adults aged between 18 to 64 (M=37.56), with minimum secondary schooling. The findings show the significant role of some factors of optimism, resilience and rumination in the prediction of subjective well-being as well as differences in self-confidence, (optimism), negative emotional experience (well-being), the ability to receive support between genders, and the tendency to experience more optimism, resilience and well-being as the individual gets older and schooling is higher. These results show how the positive features and life experience were associated with other kind of positive experiences for the well-being of individual.

Keywords: well-being; rumination; optimism; resilience; support 
Resumo: O bem-estar subjetivo abrange experiências emocionais positivas/negativas, prosperidade e satisfação com a vida. Além disso, depende de múltiplos fatores psicossociais, como: ruminação, otimismo, resiliência e capacidade de receber apoio, que foram estudados em particular e precisam ser examinados em conjunto. Para isso, este estudo se propôs a: 1) identificar o efeito das variáveis mencionadas no bemestar subjetivo de adultos e 2) explorar suas diferenças a partir do sexo, idade e escolaridade. Contou-se com a participação voluntária de 404 adultos mexicanos entre 18 e 64 anos $(\mathrm{M}=37,56)$, com escolaridade mínima de ensino médio. Os resultados mostram o papel significativo de alguns fatores de otimismo, resiliência e ruminação na predição do bem-estar subjetivo, bem como diferenças na autoconfiança (otimismo), experiência emocional negativa (bem-estar subjetivo) e capacidade de receber apoio entre os sexos, e tendência a experimentar mais otimismo, resiliência e bem-estar à medida que se tem mais idade e formação. Esses resultados mostram como os atributos positivos e a experiência de vida foram associados a outras experiências positivas para o bem-estar do indivíduo.

Palavras-chave: bem-estar; ruminação; otimismo; resiliência; apoio

Cómo citar:

Sánchez-Aragón, R. (2020). Bienestar subjetivo: el papel de la rumia, optimismo, resiliencia y capacidad de recibir apoyo. Ciencias Psicológicas, 14(2), e-2222. doi: https://doi.org/10.22235/cp.v14i2.2222

Correspondencia: Rozzana Sánchez-Aragón. Av. Universidad 3004, Col. Copilco-Universidad, C.P. 04510 Del. Coyoacán, Ciudad de México, México. E-mail: rozzara@unam.mx

La salud es concebida por la Organización Mundial de la Salud (OMS, 2006) como un estado completo de bienestar físico, espiritual, psicológico y social. Esta definición sienta las bases de la importancia que fue adquiriendo el constructo de bienestar psicológico o subjetivo (BS) (Padrós Blázquez, Gutiérrez Hernández, \& Medina Calvillo, 2015) a través del tiempo, y comprende - de acuerdo con Naci y Loannidiss (2015) y Kaufman (2016) - las elecciones y las actividades que ayudan a lograr la vitalidad física, la prontitud mental, satisfacción social, sentido de logro y la realización personal.

Hay que señalar que el BS en cierta forma representa un juicio cultural desde una perspectiva interna o creencia de las personas que pertenecen a un grupo acerca de si viven correctamente, disfrutan de la vida, es importante lo que otros piensan de cómo cada uno está viviendo la vida, así como si los individuos poseen un sentido de realización. Como puede observarse, esta noción implica por un lado, el cumplimiento de sus necesidades humanas básicas, y por el otro, los juicios éticos y evaluativos de las personas sobre sus vidas. En otras palabras, el BS refleja en cierta forma el grado en el cual las personas viven acorde a sus imperativos evolutivos y necesidades humanas, pero también representa los juicios basados en las normas y valores particulares a su cultura (Diener, 2009).

Cuando se trata de identificar los componentes del BS, hay un modelo particularmente sobresaliente, propuesto por Diener (2009). Este planteamiento es uno de los más comprehensivos e incluye: afecto positivo y afecto negativo (tiene que ver con los aspectos emocionales o afectivos experimentados por las personas), prosperidad (evalúa el éxito auto-percibido en áreas tales como relaciones, auto-estima, propósitos en la vida y optimismo) y satisfacción con la vida (se refiere a un juicio cognoscitivo respecto a la evaluación que una persona hace de la calidad de su vida en virtud de ciertos criterios como las expectativas que se tienen de ella). 
Esta investigación tomará la visión de Diener (2009) ya que su trabajo tanto conceptual como operacional ha sido más fecundo y aterrizado desde una perspectiva cultural del constructo en América Latina (e.g. Góngora \& Castro Solano, 2015; Padrós et al., 2015). Entonces, el BS se asienta en sus componentes emocionales y cognoscitivos que a su vez dependen de la calidad de una serie de dominios específicos como el contacto social, acontecimientos vitales, discrepancia entre aspiraciones y logros, percepción de auto-eficacia, así como pensamientos negativos o positivos los cuales pueden estar estrechamente vinculados con variables como la rumia que refleja malestar (Flórez Rodríguez \& Sánchez Aragón, en prensa), sus contrapartes: el optimismo que refleja bienestar y la resiliencia (Seligman, Steen, Park \& Peterson, 2005) como la capacidad para aprovechar el malestar para lograr el bienestar como se argumenta a continuación.

A la rumia se le ha definido como la tendencia a continuar pensando acerca de algo malo, doloroso o desesperanzador por un largo periodo de tiempo (Ito, Takenaka, Tomita, \& Agari, 2006). Es cuando una persona piensa repetida y recurrentemente en eventos o emociones negativas particularmente del pasado (Michael, Halligan, Clark, \& Ehlers, 2007). De hecho, se le considera como una estrategia de afrontamiento mal adaptativa que perpetúa el estrés incrementando las cogniciones negativas, deteriorando la solución de problemas y la conducta instrumental, así como reduciendo el apoyo social (Eisma \& Stroebe, 2017). Así, la rumia puede existir como un rasgo o como un estado y lo definen como un proceso psicológico perjudicial caracterizado por el pensamiento perseverante acerca de contenido negativo que genera incomodidad emocional (R. Sansone \& L. Sansone, 2012). Cabe señalar que, aunque Watkins et al. (2011) están de acuerdo con el lado perjudicial de la rumia, también reconocen que ésta puede ser un proceso psicológico benéfico ya que hay momentos en los que la rumia es específica, concreta y enfocada en el proceso lo que permite su utilidad.

Existen datos relevantes que señalan que las mujeres tienen mayor tendencia a rumiar que los hombres, lo cual contribuye a que experimenten mayor depresión, más dificultad para resolver problemas efectivamente y realizar conductas instrumentales así como mayor capacidad de corroer el apoyo social (Johnson \& Whisman, 2013; Nolen-Hoeksema, Larson, \& Grayson 1999; NolenHoeksema, Parker, \& Larson, 1994). No obstante, hay que señalar que hay evidencia de que la magnitud de la diferencia entre sexos en la rumia ha variado de estudio a estudio (Rood, Roelofs, Bogels, Nolen-Hoeksema, \& Schouten, 2009). Aunado a estos datos, la literatura señala que hay datos contradictorios respecto al comportamiento de la rumia cuando se considera la edad, es decir, autores como Nolen-Hoeksema y Aldao (2011) y Sütterlin, Paap, Babic, Kübler y Vögele (2012) dicen que a mayor edad disminuyen los pensamientos rumiantes porque las personas tienen más recursos cognoscitivos y afectivos para resolver los problemas; mientras que J. Delgado Suárez, Herrera Jiménez y Y. Delgado Suárez (2008) y García Cruz, Valencia Ortiz, Hernández Martínez y Rocha Sánchez (2017), señalan que a más edad se incrementan las ideas recurrentes como parte del proceso de envejecimiento, más aislamiento social y menos actividades. Se ha observado que, a mayor escolaridad, más diversidad de estrategias supresivas de la rumia (Delgado Suárez et al., 2008).

En cuanto a la relación entre la rumia y el BS se ha observado que la rumia es un elemento mediador entre el estrés y la capacidad de dormir (Berset, Elfering, Lüthy, Lüthi, \& Semmer, 2011; Kompier, Taris, \& Veldhoven 2012), de igual forma tiene un impacto importante en la experiencia de mayor estrés psicológico en víctimas de rompimiento amoroso (Nolen-Hoeksema, McBride \& Larson 1997). Otros hallazgos señalan que la rumia y el estrés percibido tienen una relación negativa con el bienestar psicológico que produce el ejercicio de la atención plena - mindfulness - (e.g. Deyo, Wilson, Ong, \& Koopman, 2009).

Por otro lado, se encuentran constructos como el optimismo y la resiliencia, los cuales se ubican en la literatura proveniente de la psicología positiva (Seligman et al., 2005). El primero se refiere a las expectativas positivas respecto al futuro sin importar los medios por los cuales tales resultados pueden ocurrir (Kleiman et al., 2017). Se relaciona con mayor éxito profesional, mejor 
solución de problemas, buena salud y una vida más larga (Peterson, 2000), disminuye el dolor y mejora la vitalidad (Zepeda Goncen \& Sánchez Aragón, 2019). Además, contribuye a tener mayor esperanza, auto-eficacia, motivación, confianza y perseverancia ante situaciones de gran adversidad o provocadoras de estrés (Seligman, 1991; Snyder, 1994). En congruencia, NolenHoeksema y Davis (2002), Seligman (2002) y Tashiro y Frazier (2003) sostienen que la resiliencia emerge a partir de situaciones extremadamente difíciles y altamente estresantes, permite encontrar beneficios y generar nuevos significados que favorecen el crecimiento personal y el afrontamiento de nuevos retos con mayor seguridad y eficiencia. Por lo tanto, la resiliencia es la capacidad humana para enfrentar, sobreponerse, ser fortalecido o transformado por experiencias desfavorables (Grotberg, 2003).

Al examinar los efectos del sexo sobre estas variables, se encuentra que son los hombres adolescentes quienes muestran mayor optimismo que las mujeres (Puskar et al., 2010), mientras que en un estudio de Webber y Smokowski (2018) se observó el efecto contrario; por su parte, Schwaba, Robins, Priyanka y Bleidorn (2019) evidencian la similitud entre ambos sexos respecto a la variable. En cuanto a la resiliencia, Consedine, Magai, y Krivoshekova (2005) encontraron que los hombres puntúan más alto que las mujeres, resultado apoyado por Coppari, Barcelata, Bagnoli y Codas (2018) pero con hallazgos muy débiles en una sola dimensión en muestras latinas. En Perú los hallazgos están a favor de las mujeres (Prado Álvarez \& Águila Chávez, 2003), mientras que en México, González Arratia y Valdez Medina (2013) reportan que son las mujeres quienes a través de su vida muestran más resiliencia en comparación con los hombres.

En cuanto a la edad, Londoño Pérez, Velasco Salamanca, Alejo Castañeda, Botero Soto y Vanegas (2014) indican que los adultos tienden a ser más optimistas que las personas jóvenes pues usan estrategias más constructivas y orientadas al bienestar y al éxito (Schwaba et al., 2019). En congruencia, Gooding, Hust, Johnson y Tarrier (2012) indican que los adultos fueron más resilientes - particularmente en la habilidad de regulación emocional y solución de problemasque los jóvenes, mientras que éstos últimos mostraron más resiliencia respecto al apoyo social. Y en términos de la escolaridad, Giménez Hernández (2005) y Morales Rodríguez y Díaz Barajas (2011) reportaron que a más escolaridad se puntúa más en optimismo y resiliencia (en sus factores de fortaleza, confianza en sí mismo, competencia y apoyo social) respectivamente, lo cual puede explicarse ya que estudiar provee de recursos que brindan a la persona mayores posibilidades de ser felices aún a costa de la adversidad, pueden tener más esperanza, una previsión positiva del futuro, ser más cautelosos y profundizar en el análisis de la situación que enfrentan y generar con ello un mejor enfoque y solución (Gómez Azcarate et al., 2014).

Existe evidencia de que el optimismo y la resiliencia son componentes cardinales para el BS, ya que: 1) una persona optimista espera resultados positivos aún en circunstancias difíciles, ello contribuye a que las personas se sientan mejor, tengan menos depresión y estrés y más resiliencia (Allison, Guichard, \& Gilain, 2000), 2) esto define al optimismo y a la resiliencia como factores de resistencia ante estados emocionales negativos, a la vez que 3) sirven como amortiguadores del enfrentamiento a situaciones difíciles, pues proveen de herramientas en la solución de problemas cotidianos, así como visualizar a la adversidad como un reto del cual aprender y sacar el mejor provecho. Por ello, ambos aspectos impactan directamente en el BS propio, pues ahí se refleja la evaluación de los propios recursos, del manejo óptimo de las situaciones, lo cual en consecuencia afecta el auto-estima, la auto-eficacia y la salud (Chopik, Kim, \& Smith, 2018).

Pero la resiliencia como recurso personal para salir adelante puede combinarse con un recurso social de alto valor en la vida del individuo: el apoyo social (Sullivan \& Davila, 2010). Mismo que tiende a mejorar al ajuste psicológico y la salud (Sarason et al., 1983), la auto-estima (sentirse aceptado y valorado), el estado de ánimo positivo y un punto de vista favorable sobre la vida, predice BS y modera el impacto del estrés en la vida (Procidano, 1992). Aunado a lo anterior, el apoyo es un factor de protección que provee de recursos psicológicos necesarios para enfrentar 
el estrés (Chi et al., 2011) y de conectarse con otros significativos que pueden resultar de gran ayuda; no obstante, éste puede resultar malogrado si el propio individuo no tiene la capacidad de recibirlo (Sánchez Aragón, en prensa; Verhofstadt, Lemmens, \& Buysse, 2013).

Así, los objetivos de este estudio fueron: 1) identificar el efecto de la rumia, el optimismo, la resiliencia y la capacidad de recibir apoyo en el BS en adultos mexicanos, y 2) explorar si el sexo, la edad y la escolaridad producen diferencias en las variables antes mencionadas.

\section{Método}

\section{Participantes}

Se trabajó con una muestra no probabilística (Hernández Sampieri, Fernández Collado, \& Baptista Lucio, 2014) de 404 personas (202 mujeres y 202 hombres) mexicanas de entre 18 y 64 años $(M=37.56)$, con escolaridad de secundaria $(18.6 \%)$, preparatoria $(31.9 \%)$ y licenciatura (48\%) y que tenían de tiempo en la relación con su pareja entre 5 meses y 41 años $(M=14.21$ años). El estado civil reportado fue de unión libre (45.8\%), casados $(54.2 \%)$ y en cuanto al número de hijos hubo una variedad desde 0 hasta 5 (Moda=2). Cabe señalar que para cumplir con el segundo objetivo fue necesario crear tres grupos de edad que fueran equivalentes en cuanto a la cantidad de participantes y comparables a la vez que resultaran congruentes con ciertas etapas del proceso de vida: 17 a 30 años (grupo a), 31 a 44 (grupo b) y 45 a 62 (grupo c).

\section{Instrumentos}

Cuestionario de Datos Sociodemográficos, que se compone de algunas preguntas que permiten describir a la muestra, así como clasificarlos para algún fin estadístico. Las preguntas incluidas fueron: sexo, edad, escolaridad, tiempo en la relación con su pareja, estado civil y número de hijos.

Escala de Rumia (Flórez Rodríguez \& Sánchez Aragón, en prensa) que consta -en su versión corta- de 10 reactivos con formato de respuesta tipo Likert de cinco puntos que indican grados de acuerdo, distribuidos en dos factores: 1) malestar ("Le doy vuelta y vuelta a mi sufrimiento") ( $\alpha=.89)$ y 2 ) reflexión obsesiva ("Pienso todo el tiempo en todas mis deficiencias, carencias, defectos y errores) $(\alpha=.84)$.

Escala de Optimismo (Sánchez Aragón, 2018) en su versión corta comprende 20 reactivos en formato tipo Likert de cinco puntos que indican grados de acuerdo y que se organizan en cuatro factores: 1) actitud positiva ("Soy optimista aunque parezca que lo que viene va a ser negativo") $(\alpha=.90), 2)$ control interno ("Creo que si tu trabajas lo suficientemente duro, podrás lograr cualquier cosa") $(\alpha=.78), 3)$ auto-confianza ("Ninguna tarea es demasiado difícil para mí") $(\alpha=.81)$ y 4) esperanza ("Creo que mi futuro va a estar muy bien") $(\alpha=.80)$.

Escala de Resiliencia (Palomar Lever \& Gómez Valdez, 2010) con 25 reactivos en formato tipo Likert (versión corta) que forman en cinco factores: 1) fortaleza y confianza en sí mismo ("Lo que me ha ocurrido en el pasado me hace sentir confianza para enfrentar nuevos retos") $(\alpha=.92), 2)$ competencia social ("Me es fácil establecer contacto con otras personas") $(\alpha=.87), 3)$ apoyo familiar ("Tengo una buena relación con mi familia") $(\alpha=.87), 4)$ apoyo social (“Tengo algunos amigos/familia...que realmente se preocupan por mí") $(\alpha=.84)$ y, 5) estructura ("Las reglas y la rutina hacen mi vida más fácil") $(\alpha=.79)$.

Escala de Recepción de Apoyo (Sánchez Aragón, en prensa) que consta de 7 reactivos Likert $(\alpha=.79)$ en un solo factor: "Comparto mis sentimientos con otras personas para ver si me ayudan", "Dejo que las personas cercanas a mí me brinden su apoyo", "Estoy dispuesto a recibir consejos cuando me enfrento a una situación difícil". 
Escalas de Bienestar Subjetivo (Diener, Emmons, Larsen, \& Griffin, 1985; Diener, 2009; Granillo Velasco, Sánchez Aragón, \& Zepeda Goncen, 2020):

1. Experiencias Emocionales con 12 reactivos Likert en dos factores: 1) positiva ("Placer", "Felicidad") ( $\alpha=.85)$ y 2) negativa ("Tristeza", "Mal") $(\alpha=.81)$.

2. Prosperidad con 8 reactivos Likert en un factor: "Soy una buena persona y vivo una buena vida", "Tengo una vida útil y significativa" $(\alpha=.89)$.

3. Satisfacción de Vida con 5 reactivos Likert en un factor: "Estoy satisfecho con mi vida", "Las circunstancias de mi vida son buenas" $(\alpha=.84)$.

\section{Procedimiento}

La aplicación de aproximadamente 20 minutos se llevó a cabo por psicólogos calificados que acudieron a lugares donde se podían encontrar a personas con una relación de pareja actual y con mínimo un mes de vivir juntos (plazas comerciales, casas, escuelas, oficinas, centros recreativos y culturales, etc.), para que contestaran en forma voluntaria, anónima las escalas (ordenadas siempre en el mismo orden) en cuestión, haciéndoles saber que sus datos eran confidenciales, que no les causarían daños y solo se utilizarían con fines científicos. Asimismo, se respondió inmediatamente a sus dudas y dejando a disposición de los participantes sus resultados personales.

\section{Análisis de Datos}

Con el propósito de responder a los objetivos de esta investigación se realizó en primera instancia un análisis de Kolmogorov-Smirnov para explorar si las variables tenían una distribución normal, y con base en ello se decidió realizar una regresión logística binaria para responder al primero objetivo, posteriormente para comparar los grupos se utilizaron tanto la prueba $U$ de Mann Whitney como la prueba de Kruskal Wallis. Para ello se trabajó con el programa SPSS (Statistical Program for Social Sciences) versión 23.

\section{Resultados}

Como se mencionó previamente, se realizó la prueba de Kolmogorov-Smirnov cuyo fin es identificar si las variables se distribuyen normalmente. En ninguno de los casos se cumplió esta condición ( $p<=.041)$ por lo que la elección de los análisis estadísticos se basó en estos resultados.

Con el propósito de responder al primer objetivo de la presente investigación, se realizaron inicialmente algunos análisis de regresión lineal para probar los supuestos de independencia de errores y no multicolinealidad de las variables. En cuanto al primer supuesto se encontró que para las variables dependientes se identificaron puntajes de Durbin-Watson entre 1 y 3 lo que cumple la independencia de errores (experiencia emocional positiva $=1.933$, negativa $=1.888$, prosperidad $=1.906$ y satisfacción de vida $=1.822$ ).

Respecto al segundo supuesto, se encontraron coeficientes que indicaron multicolinealidad, por lo que se tomó la decisión de realizar un análisis factorial de segundo orden y con ello poder cumplir con este requisito. Las agrupaciones de factores quedaron como sigue: factor 1 integró todos los factores de optimismo, factor 2 se compuso de todos los factores de resiliencia aunado a la capacidad para recibir apoyo (nombrada desde este momento resiliencia+) y finalmente, el factor 3 se formó con los dos factores de rumia. Ya habiendo realizado estos análisis, se realizaron las regresiones logísticas donde las variables dependientes fueron: experiencia emocional positiva, experiencia emocional negativa, prosperidad y satisfacción de vida; y las variables predictoras: optimismo, resiliencia+ y rumia. 
Experiencia Emocional Positiva. Para el análisis de regresión logística el bloque cero indica que hay un $89 \%$ de probabilidad de acierto en el resultado de la variable dependiente asumiendo que la mayor parte de las personas experimentan emociones positivas altas. Para el bloque uno del modelo la puntuación de eficiencia estadística de ROA indica que hay una mejora significativa en la predicción de la probabilidad de ocurrencia de la experiencia emocional positiva $\left(X^{2}=44.649 ; \mathrm{gl}=3 ; p=.000\right) . \mathrm{La} \mathrm{R}^{2}$ de Nagelkerke indica que el modelo propuesto explica el $23.7 \%$ de la varianza de la variable dependiente en cuestión (0.237). No obstante, la prueba de Hosmer y Lemeshow indica que la varianza explicada por el modelo explica un porcentaje no significativo de varianza $(0.522)$.

Para el análisis de regresión logística el bloque uno indica que hay un $89.8 \%$ de probabilidad de acierto en el resultado de la variable dependiente (experiencia emocional positiva) cuando se ha integrado a la predicción la rumia, el optimismo y la resiliencia. Y se observa que a medida que aumenta el puntaje del optimismo, aumenta más la experiencia emocional positiva. La puntuación para el modelo probado indica que la variable de optimismo aporta significativamente a la predicción de la variable dependiente (experiencia emocional positiva). Los resultados obtenidos de este modelo se pueden generalizar a la muestra (ver Tabla 1).

Tabla 1

Predicción de la Probabilidad de ocurrencia de la Experiencia Emocional Positiva

\begin{tabular}{lllllll}
\hline & $B$ & $\begin{array}{l}\text { Error } \\
\text { estándar }\end{array}$ & Wald & $g l$ & $p$ & Exp(B) \\
\hline Rumia & -0.405 & 0.221 & 3.343 & 1 & .068 & 0.600 \\
Optimismo & 1.636 & 0.464 & 12.436 & 1 & .000 & 5.133 \\
Resiliencia & 0.513 & 0.430 & 1.426 & 1 & .232 & 1.671 \\
\hline Constante & -5.156 & 1.910 & 7.284 & 1 & .007 & 0.006 \\
\hline
\end{tabular}

Experiencia Emocional Negativa. Para el análisis de regresión logística el bloque cero indica que hay un $86 \%$ de probabilidad de acierto en el resultado de la variable dependiente asumiendo que la mayor parte de las personas sienten experiencia emocional negativa alta. Para el bloque uno del modelo la puntuación de eficiencia estadística de ROA indica que hay una mejora significativa en la predicción de la probabilidad de ocurrencia de la experiencia emocional negativa $\left(X^{2=} 56.966 ; \mathrm{gl}=3 ; p=.000\right)$. La $\mathrm{R}^{2}$ de Nagelkerke indica que el modelo propuesto explica el $20.7 \%$ de la varianza de la variable dependiente bajo estudio (.207). No obstante, la prueba de Hosmer y Lemeshow indica que la varianza explicada por el modelo explica un porcentaje no significativo de varianza (0.755). Para el análisis de regresión logística el bloque uno indica que hay un $86.6 \%$ de probabilidad de acierto en el resultado de la variable dependiente (experiencia emocional negativa) cuando se ha integrado a la predicción la rumia, el optimismo y la resiliencia. Y se observa que a medida que aumenta el puntaje de la rumia, aumenta la probabilidad de experiencia emocional negativa y que, a más optimismo y resiliencia, disminuye dicha probabilidad. La puntuación para el modelo probado indica que las variables de rumia, optimismo y resiliencia, aportan significativamente a la predicción de la variable dependiente (experiencia emocional negativa). Los resultados obtenidos de este modelo se pueden generalizar a la muestra (ver Tabla 2). 
Tabla 2

Predicción de la Probabilidad de ocurrencia de la Experiencia Emocional Negativa

\begin{tabular}{lllllll}
\hline & $B$ & $\begin{array}{l}\text { Error } \\
\text { estándar }\end{array}$ & Wald & $g l$ & $p$ & $\operatorname{Exp}(B)$ \\
\hline Rumia & 0.928 & 0.214 & 18.897 & 1 & .000 & 2.530 \\
Optimismo & -0.869 & 0.393 & 4.899 & 1 & .027 & 0.419 \\
Resiliencia & -0.779 & 0.390 & 3.998 & 1 & .046 & 0.459 \\
\hline Constante & 2.272 & 1.663 & 1.865 & 1 & .172 & 9.694 \\
\hline
\end{tabular}

Prosperidad. Para el análisis de regresión logística el bloque cero indica que hay un $96.6 \%$ de probabilidad de acierto en el resultado de la variable dependiente asumiendo que la mayor parte de las personas sienten prosperidad alta. Para el bloque uno del modelo la puntuación de eficiencia estadística de ROA indica que hay una mejora significativa en la predicción de la probabilidad de ocurrencia de la prosperidad $\left(X^{2=} 35.386 ; \mathrm{gl}=3 ; p=.000\right)$. La $\mathrm{R}^{2}$ de Nagelkerke indica que el modelo propuesto explica el $37.0 \%$ de la varianza de la variable prosperidad (.370). No obstante, la prueba de Hosmer y Lemeshow indica que la varianza explicada por el modelo explica un porcentaje no significativo de varianza (0.816). Para el análisis de regresión logística el bloque uno indica que hay un $96.4 \%$ de probabilidad de acierto en el resultado de la variable dependiente (prosperidad) cuando se ha integrado a la predicción la rumia, el optimismo y la resiliencia. Y se observa que a medida que aumenta el puntaje de la rumia, disminuye la prosperidad y que, a más optimismo y resiliencia, incrementa la probabilidad de prosperidad. La puntuación para el modelo probado indica que las variables de rumia, optimismo y resiliencia, aportan significativamente a la predicción de la variable dependiente (prosperidad). Los resultados obtenidos de este modelo se pueden generalizar a la muestra (ver Tabla 3 ).

Tabla 3

Predicción de la Probabilidad de ocurrencia de la Prosperidad

\begin{tabular}{lllllll}
\hline & $B$ & $\begin{array}{l}\text { Error } \\
\text { estándar }\end{array}$ & Wald & $g l$ & $p$ & $\operatorname{Exp}(B)$ \\
\hline Rumia & -0.405 & 0.221 & 3.343 & 1 & .068 & 0.600 \\
Optimismo & 1.636 & 0.464 & 12.436 & 1 & .000 & 5.133 \\
Resiliencia & 0.513 & 0.430 & 1.426 & 1 & .232 & 1.671 \\
\hline Constante & -5.156 & 1.910 & 7.284 & 1 & .007 & 0.006 \\
\hline
\end{tabular}

Satisfacción de Vida. Para el análisis de regresión logística el bloque cero indica que hay un $90.2 \%$ de probabilidad de acierto en el resultado de la variable dependiente asumiendo que la mayor parte de las personas sienten satisfacción de vida alta. Para el bloque uno del modelo la puntuación de eficiencia estadística de ROA indica que hay una mejora significativa en la predicción de la probabilidad de ocurrencia de la satisfacción de vida $\left(X^{2=} 56.458 ; \mathrm{gl}=3 ; p=.000\right)$. 
La $\mathrm{R}^{2}$ de Nagelkerke indica que el modelo propuesto explica el $30.9 \%$ de la varianza de la variable satisfacción de vida (.309). No obstante, la prueba de Hosmer y Lemeshow indica que la varianza explicada por el modelo explica un porcentaje no significativo de varianza (0.440). Para el análisis de regresión logística el bloque uno indica que hay un $90.5 \%$ de probabilidad de acierto en el resultado de la variable dependiente (satisfacción de vida) cuando se ha integrado a la predicción la rumia, el optimismo y la resiliencia. Y se observa que a medida que aumenta el puntaje de la rumia, disminuye la satisfacción de vida y que, a más optimismo, aumenta la probabilidad de que se sienta más satisfacción de vida. La puntuación para el modelo probado indica que las variables de rumia y optimismo, aportan significativamente a la predicción de la variable dependiente. Los resultados obtenidos de este modelo se pueden generalizar a la muestra (ver Tabla 4).

Tabla 4

Predicción de la Probabilidad de ocurrencia de la Satisfacción de Vida

\begin{tabular}{lllllll}
\hline & $B$ & $\begin{array}{l}\text { Error } \\
\text { estándar }\end{array}$ & Wald & $g l$ & $p$ & $\operatorname{Exp}(B)$ \\
\hline Rumia & -0.511 & 0.238 & 4.603 & 1 & .032 & 0.600 \\
Optimismo & 2.213 & 0.544 & 16.532 & 1 & .000 & 9.145 \\
Resiliencia & 0.361 & 0.484 & 0.558 & 1 & .455 & 1.435 \\
\hline Constante & -6.261 & 2.147 & 8.505 & 1 & .004 & 0.002 \\
\hline
\end{tabular}

En cuanto al segundo objetivo de este estudio, se realizaron análisis comparativos entre grupos con base en el sexo, edad y escolaridad. Para el primer caso se hizo la prueba de $U$ de Mann Whitney que señala la supremacía de las mujeres re specto a la experiencia negativa (BS) y la capacidad para recibir apoyo, mientras que los hombres puntuaron más alto en auto-confianza. En los otros casos no se observaron diferencias estadísticamente significativas (ver Tabla 5). 
Tabla 5.

Diferencias entre hombres y mujeres

\begin{tabular}{|c|c|c|c|c|c|}
\hline & & Mujeres & Hombres & $z$ & $p$ \\
\hline \multirow{2}{*}{ 营 } & Malestar & 201.11 & 185.89 & -1.344 & .179 \\
\hline & Reflexión Obsesiva & 201.51 & 183.49 & -1.598 & .110 \\
\hline \multirow{4}{*}{ 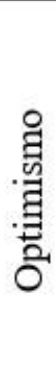 } & Actitud Positiva & 187.73 & 199.27 & -1.023 & .306 \\
\hline & Control Interno & 185.54 & 200.42 & -1.326 & .185 \\
\hline & Auto-confianza & 181.04 & 203.84 & -2.021 & .043 \\
\hline & Esperanza & 194.15 & 189.86 & -.380 & .704 \\
\hline \multirow{5}{*}{ 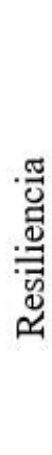 } & Fortaleza & 191.54 & 194.46 & -.259 & .796 \\
\hline & Competencia & 190.25 & 191.76 & -.134 & .893 \\
\hline & Apoyo familiar & 190.88 & 194.09 & -.286 & .775 \\
\hline & Apoyo Social & 199.98 & 185.98 & -1.267 & .205 \\
\hline & Estructura & 193.60 & 188.36 & -.466 & .641 \\
\hline \multirow{5}{*}{  } & Experiencia Positiva & 197.65 & 183.43 & -1.269 & .205 \\
\hline & Experiencia Negativa & 203.81 & 175.34 & -2.540 & .011 \\
\hline & Prosperidad & 189.66 & 195.28 & -.498 & .619 \\
\hline & Satisfacción de vida & 194.47 & 191.54 & -.259 & .795 \\
\hline & $\begin{array}{c}\text { Capacidad de Recibir } \\
\text { Apoyo }\end{array}$ & 204.39 & 171.70 & -2.925 & .003 \\
\hline
\end{tabular}

En tanto, para examinar las diferencias por edad y por escolaridad se realizaron algunas pruebas de Kruskal Wallis. Con base en la edad, se identificaron algunas diferencias estadísticamente significativas que muestran que el grupo de 17 a 30 años presenta más rumia y experiencia negativa (BS) en comparación con los otros grupos, el grupo de 31 a 44 años puntúan más en apoyo familiar y estructura (resiliencia) en comparación con el grupo más joven, mientras que el grupo de 45 a 62 años tienen una actitud más positiva y auto-confianza (optimismo), fortaleza (resiliencia) así como mayor prosperidad en comparación con los más jóvenes (ver Tabla $6)$. 
Tabla 6.

Diferencias por edad

\begin{tabular}{|c|c|c|c|c|c|c|}
\hline & & $17-30 a$ & $31-44 b$ & $45-62 c$ & $X^{2}$ & $p$ \\
\hline \multirow{2}{*}{ 茎 } & Malestar & 220.36 & 186.77 & 171.62 & 12.602 & .002 \\
\hline & Reflexión Obsesiva & 222.68 & 176.89 & 177.42 & 14.385 & .001 \\
\hline \multirow{4}{*}{ 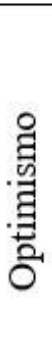 } & Actitud Positiva & 171.62 & 189.41 & 218.96 & 11.559 & .003 \\
\hline & Control Interno & 185.88 & 186.71 & 205.78 & 2.616 & .270 \\
\hline & Auto-confianza & 164.98 & 195.10 & 216.23 & 13.513 & .001 \\
\hline & Esperanza & 195.30 & 175.73 & 205.54 & 4.930 & .085 \\
\hline \multirow{5}{*}{ 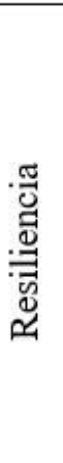 } & Fortaleza & 173.52 & 194.78 & 209.51 & 6.621 & .036 \\
\hline & Competencia & 177.55 & 197.31 & 196.33 & 2.611 & .271 \\
\hline & Apoyo familiar & 170.23 & 203.03 & 201.73 & 7.223 & .027 \\
\hline & Apoyo Social & 190.90 & 201.07 & 184.30 & 1.597 & .450 \\
\hline & Estructura & 167.83 & 200.97 & 202.08 & 7.913 & .019 \\
\hline \multirow{6}{*}{ 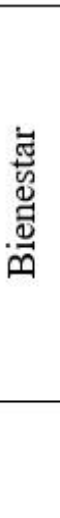 } & Experiencia Positiva & 194.96 & 188.59 & 186.40 & .407 & .816 \\
\hline & Experiencia Negativa & 222.59 & 176.98 & 167.29 & 18.151 & .000 \\
\hline & Prosperidad & 175.65 & 188.50 & 213.05 & 7.265 & .026 \\
\hline & Satisfacción de vida & 185.50 & 186.91 & 206.27 & 2.718 & .257 \\
\hline & Capacidad de Recibir & 180.99 & 189.47 & 191.79 & .672 & .714 \\
\hline & Apoyo & & & & & \\
\hline
\end{tabular}

Y finalmente, se encontró que la muestra de secundaria puntuó más en reflexión obsesiva (rumia) en comparación con la de licenciatura principalmente. Y estos mismos participantes (licenciatura) puntuaron más en todos los factores de resiliencia y optimismo, prosperidad y capacidad de recibir apoyo en comparación con aquellos con estudios de secundaria (ver Tabla 7). 
Tabla 7

Diferencias por Escolaridad

\begin{tabular}{|c|c|c|c|c|c|c|}
\hline & & Secundaria & Preparatoria & Licenciatura & $X^{2}$ & $p$ \\
\hline \multirow{2}{*}{ 湆 } & Malestar & 206.19 & 189.93 & 184.80 & 1.988 & .370 \\
\hline & Reflexión Obsesiva & 215.89 & 192.91 & 177.12 & 6.698 & .035 \\
\hline \multirow{4}{*}{ 营 } & Actitud Positiva & 158.77 & 184.44 & 208.08 & 11.301 & .004 \\
\hline & Control Interno & 156.08 & 187.85 & 204.71 & 10.478 & .005 \\
\hline & Auto-confianza & 158.05 & 187.52 & 202.96 & 8.791 & .012 \\
\hline & Esperanza & 160.47 & 189.50 & 199.41 & 6.561 & .038 \\
\hline \multirow{5}{*}{ 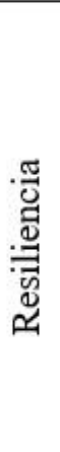 } & Fortaleza & 159.25 & 189.69 & 202.41 & 8.193 & .017 \\
\hline & Competencia & 129.39 & 195.27 & 205.87 & 26.464 & .000 \\
\hline & Apoyo familiar & 164.09 & 179.97 & 205.62 & 8.995 & .011 \\
\hline & Apoyo Social & 151.86 & 179.70 & 211.61 & 18.082 & .000 \\
\hline & Estructura & 151.61 & 185.59 & 203.24 & 11.717 & .003 \\
\hline \multirow{6}{*}{ 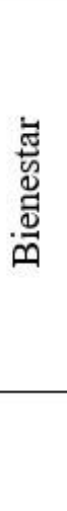 } & Experiencia Positiva & 203.32 & 192.57 & 177.95 & 3.243 & .198 \\
\hline & Experiencia Negativa & 194.88 & 194.13 & 178.15 & 2.140 & .343 \\
\hline & Prosperidad & 157.16 & 181.61 & 207.39 & 11.977 & .003 \\
\hline & Satisfacción de vida & 171.63 & 190.15 & 196.91 & 2.757 & .252 \\
\hline & Capacidad de Recibir & 154.45 & 178.40 & 202.84 & 11.322 & .003 \\
\hline & Apoyo & & & & & \\
\hline
\end{tabular}

\section{Discusión}

Los objetivos de esta investigación fueron: 1) identificar el efecto de la rumia, el optimismo, la resiliencia y la capacidad de recibir apoyo en el BS en adultos mexicanos, y 2) explorar si el sexo, la edad y la escolaridad producen diferencias en las variables antes mencionadas.

Para cumplir con el primer propósito, se realizaron algunos análisis de regresión logística que exigieron la realización de un análisis factorial de componentes principales que unificó a los factores de cada una de las variables predictoras (rumia, optimismo y resiliencia) y sumando la capacidad para recibir apoyo a la última. Estos análisis permitieron identificar -a partir de los 
predictores mencionados-, la probabilidad de ocurrencia de cada uno de los componentes del modelo de bienestar subjetivo planteado por Diener (2009) y estudiado cuantiosamente alrededor del mundo. Así, se observó que para que se presente la experiencia emocional positiva alta (como el placer, la felicidad o gozo) en los participantes es debido a la contribución del optimismo. Esto puede deberse a que las sensaciones satisfactorias — como las antes mencionadas - son más factibles cuando se posee una disposición más positiva hacia eventos del futuro, se considera que a partir de las propias acciones se puede estar bien, se siente auto-confianza y esperanza de lo que puede pasar en lo inmediato y mediato (Seligman, 1991; Snyder, 1994). En congruencia, para que se pueda predecir un incremento en la ocurrencia de la experiencia de emociones negativas (como miedo, displacer o tristeza) es debido a un aumento en la rumia así como en una disminución del optimismo, la resiliencia y la capacidad de recibir apoyo. Esto puede darse ya que tanto en el malestar como en la reflexión obsesiva que se vive, genera incomodidad emocional (R. Sansone \& L. Sansone, 2012) que perjudica a la persona. Además, el ser pesimista y en consecuencia tener pensamientos y emociones alrededor del disgusto, fastidio, pesadumbre y sufrimiento pasados, permean la cotidianidad con que perpetúan el estrés y el bajo desempeño psicosocial minando de manera importante la capacidad necesaria para salir adelante y el apoyo social (Eisma \& Stroebe, 2017), lo que contradice a Watkins et al. (2011) cuando señala que la rumia tiene una arista positiva, misma que deberá examinarse más cuidadosamente.

Por otro lado, la actitud positiva, el control interno, la auto-confianza y la esperanza (optimismo), predice la probabilidad de que se produzca una sensación de alta prosperidad en los participantes. De acuerdo a Diener (2009) la prosperidad es una sensación de éxito personal, de sus relaciones personales y propósitos en la vida, que se nutre y es nutrido por otros atributos personales que facilitan y dirigen la vida - como el optimismo- y brindando bienestar psicológico.

La satisfacción de vida puede ocurrir — de acuerdo a los hallazgos de esta investigacióncuando se tiene disminución de la rumia, es decir, pensamientos obsesivos que generan malestar y aumento del optimismo (e.g. ser positivo aunque parezca que lo que viene va a ser negativo, creer que si se trabaja lo suficientemente duro se puede lograr cualquier cosa, creer que ninguna tarea es demasiado difícil y que el futuro es prometedor). Esto resulta evidente ya que el juicio global de bienestar descansa en la evaluación que una persona hace de la calidad de su vida en virtud de ciertos criterios como las expectativas que se tienen de ella, y el optimismo está representado precisamente por la esperanza de que lo que viene es superable y positivo no importando lo que sea necesario hacer (Kleiman et al., 2017), lo que automáticamente explica el papel de la rumia.

Respecto al segundo objetivo, los datos muestran que en términos de rumia, resiliencia, experiencia emocional positiva, prosperidad y satisfacción de vida se observaron similitudes más que diferencias, es decir, tanto hombres como mujeres tienden a poseer — en igual medidapensamientos repetitivos y recurrentes sobre emociones negativas experimentadas en el pasado (Ito et al., 2006; Michael et al., 2007) y capacidad para enfrentar, sobreponerse, ser fortalecido o transformado por experiencias desfavorables (Grotberg, 2003), tener experiencias emocionales como el gozo y la felicidad así como sentirse exitoso en la vida y sentir que su vida ha sido buena. No obstante, sí se observaron algunas diferencias estadísticamente significativas que señalan que las mujeres mostraron mayor experiencia negativa en el último mes y más capacidad de recibir apoyo; mientras que los hombres mostraron más auto-confianza (factor de optimismo). Esto podría deberse a que son ellas quienes han sido identificadas como más sensibles emocionalmente abarcando las emociones negativas, es decir, dado que han desarrollado más habilidades en este terreno, sus vivencias y expresiones son más intensas y expresivas (e.g. Feldman-Barret, Lane, Sechrest, \& Schwartz, 2000; López Usero, 2016). Aunado a ello, se ha observado que son las mujeres quienes suelen establecer relaciones personales más cercanas (Juárez Ramírez, Valdez Santiago, \& Hernández Rosete, 2005) en donde tienden a compartir más de su vida y emociones, 
evidenciando con eso su disposición a recibir ayuda, consejo, etc. (Matud, Ibañez, Bethencourt, Marrero, \& Carballeira, 2003; Pettus-Davis, Veeh, Davis, \& Tripodi, 2018).

Cuando se examinaron las posibles diferencias en la rumia, optimismo, resiliencia, BS y capacidad de apoyo por edad, se encontraron algunas diferencias estadísticamente significativas que muestran que el grupo de 17 a 30 años presenta más rumia y experiencia negativa (BS) en comparación con los grupos mayores. Esto podría deberse a que en la juventud las personas suelen apreciar la vida de tal manera que sienten el malestar de sus errores o emociones del pasado que pueden haberse generado por la inexperiencia, a diferencia de los grupos mayores quienes pueden encontrar lo positivo en los pequeños detalles de la vida (Reig, 2000). Estos resultados son congruentes con lo señalado por Nolen-Hoeksema y Aldao (2011) y Sütterlin et al. (2012) quienes explican esto con base en la idea de que conforme pasan los años, las personas van adquiriendo más recursos cognoscitivos y afectivos para resolver problemas. Asimismo, se observó que el grupo de 31 a 44 años puntúa más en apoyo familiar y estructura en comparación con el grupo más jóven, lo cual resulta lógico — y afín con el resultado previo - ya que conforme se tiene más edad se van adquiriendo más orden y organización en la vida que permiten ante momentos de estrés, mantenerse ocupado y manejar más la situación. Esto en conjunto con las redes de apoyo que se van teniendo, proveen a los individuos de protección en momentos de necesidad (Jiang, Drolet, \& Kim, 2018). Por último, el grupo de 45 a 62 años tiene una actitud más positiva, auto-confianza y fortaleza, así como mayor prosperidad en comparación con los más jóvenes, lo cual se ve apoyado por Londoño et al. (2014) y Schawaba et al. (2019) quienes indican que a más edad se es más optimista, se valoran más las relaciones con otros, se cuenta con y usan estrategias más constructivas y orientadas al bienestar, lo que permite obtener más logros en diferentes esferas de la vida lo que les permite sentirse más prósperos y exitosos en la vida.

Finalmente, se tuvieron dos hallazgos principales al explorar posibles diferencias en las variables bajo estudio por escolaridad. Así, por un lado, se encontró que la muestra de secundaria puntuó más en reflexión obsesiva en comparación con la de licenciatura principalmente, lo cual puede deberse a que menos educación hay menos capacidades de suprimir este tipo de pensamientos (Delgado Suárez et al., 2008), máxime que posiblemente se han experimentado desafíos más difíciles de resolver por la falta de experiencia y capacidad. Y, por otro lado, precisamente quienes cuentan con estudios de licenciatura, puntuaron más en todos los factores de optimismo y resiliencia, prosperidad y capacidad de recibir apoyo en comparación con aquellos con estudios de secundaria. Y es que a través de la educación estudiar provee de recursos que brindan a la persona mayores posibilidades de ser felices aún a costa de la adversidad, pueden tener más esperanza, una previsión positiva del futuro, ser más cautelosos y profundizar en el análisis de la situación que enfrentan y generar con ello un mejor enfoque y solución (Giménez Hernández, 2005; Gómez Azcarate et al., 2014; Morales Rodríguez \& Díaz Barajas, 2011). De la misma forma se desarrollan más relaciones personales que sensibilizan a la persona para que pueda reconocer que se necesita de otros. Todo en su conjunto guía a la idea de que estos recursos que brindan los estudios proveen de prosperidad material y psicológica (Carmona Valdés, 2009; García \& Hoffman, 2002).

En conclusión, se puede decir que el BS es producto de algunos aspectos vinculados principalmente al optimismo y al pensamiento rumiante. Aunado a ello que la edad y escolaridad - más que el sexo- afectan de manera importante la experiencia de estas variables, lo que debe tomarse en cuenta para futuras investigaciones. Se considera que este estudio logró escudriñar en la relación a la vez que diferencias en el comportamiento de estas variables desde un ángulo más amplio y en una muestra de 404 participantes, lo que resulta en una contribución a su entendimiento de los constructos y de este grupo. 


\section{Referencias}

Allison P. J., Guichard C. \& Gilain L. (2000). A prospective investigation of dispositional optimism as a predictor of health-related quality of life in head and neck cancer patients. Quality of life research: an international journal of quality of life aspects of treatment, care and rehabilitation, 9(8) 951-60. doi: 10.1023/A:1008931906253

Berset, M., Elfering, A., Lüthy, S., Lüthi, S., \& Semmer, N. K. (2011). Work stressors and impaired sleep: rumination as a mediator. Stress \& Health, 27(2), 71-82. doi: 10.1002/smi.1337

Carmona Valdés, S. E. (2009). El bienestar personal en el envejecimiento. Revista de Ciencias Sociales de la Universidad Iberoamericana, IV(7), 48-65.

Chi, P., Tsang, S. K. M., Chan, K. S., Xiang, X., Yip, P. S. F., Cheung, Y. T., \& Zhang, X. (2011). Marital satisfaction of Chinese under stress: Moderating effects of personal control and social support. Asian Journal of Social Psychology, 14, 15-25. doi: 10.1186 / 1471-2458-13-1150

Chopik, W. J., Kim, E. S., \& Smith, J. (2018). An examination of dyadic changes in optimism and physical health over time. Health Psychology, 37(1), 42-50. doi: 10.1037 / hea0000549

Consedine, N. S., Magai, C., \& Krivoshekova, Y. S. (2005). Socioemotional functioning in older adults and their links to physical resilience. Ageing International, 30(3), 209-244. doi: 10.1007/s12126-005-1013-z

Coppari, N., Barcelata B. E., Bagnoli, L., \& Codas, G. (2018). Efectos de la edad, el sexo y el contexto cultural en la disposición resiliente de los adolescentes de Paraguay y México. Revista de Psicología Clínica con Niños y Adolescentes, 5(1), 16-22. doi: 10.21134/rpcna.2018.05.1.2

Delgado Suárez, J., Herrera Jiménez, L., \& Delgado Suárez, Y. (2008). La mediatización del pensamiento rumiativo en el accidente cerebrovascular. Revista de la Facultad de Ciencias de la Salud, 5(1), 15-23.

Deyo, M., Wilson, K. A., Ong, J. \& Koopman, C. (2009). Mindfulness and rumination: does mindfulness training lead to reductions in the ruminative thinking associated with depression? Explore, 5(5), 265-271.

Diener, E. (2009). Culture and Well-being Works of Ed Diener. En E. Diener (Ed.), Culture and Well-being: The Collected Works (pp. 1-8). New York: Springer.

Diener, E., Emmons, R., Larsen, R., \& Griffin, S. (1985). The Satisfaction with Life Scale. Journal of Personality Assessment, 49(1), 71-75. doi: 10.1207/s15327752jpa4901_13

Eisma, M. C. \& Stroebe, M. S. (2017). Rumination following bereavement: an overview. Bereavement Care, 36(2), 58-64. doi: 10.1080/02682621.2017.1349291

Feldman-Barret, L., Lane, R. D., Sechrest, L., \& Schwartz, G. E. (2000). Sex differences in emotional awareness. Personality and Social Psychology Bulletin, 26(9), 1027-1035. doi: $10.1177 / 01461672002611001$

Flórez Rodríguez, Y. N. \& Sánchez Aragón, R. (en prensa). Evaluando el amor compasivo en la pareja. Salud y Administración.

García, P. S. \& Hoffman, S. T. (2002). El Bienesar como preferencia y las mediciones de pobreza. Cinta Moebio, 13, 70-73. 
García Cruz, R., Valencia Ortiz, A. I., Hernández Martínez, A., \& Rocha Sánchez, T. E. (2017). Pensamiento rumiativo y depresión entre estudiantes universitarios: repesando el impacto del género. Interamerican Journal of Psychology, 51(3), 406-416.

Giménez Hernández, M. (2005). Optimismo y pesimismo: variables asociadas en el contexto escolar. Pulso, 28, 9-23.

Gómez Azcarate, E., Vera, A., Ávila, M. E., Musitu, G., Vega, E., \& Dorantes, G. (2014). Resiliencia y felicidad en adolescentes frente a la marginación urbana en México. Psicodebate, 14(1), 45-68. doi: 10.18682/pd.v14i1.334

Góngora, V. C., \& Castro Solano, A. (2015). La validación de un índice de bienestar para población adolescente y adulta de la ciudad de Buenos Aires. PSIENCIA. Revista Latinoamericana de Ciencia Psicológica, 7, 329-338. doi: 10.5872/psiencia/7.2.21

González Arratia López Fuentes, N. I. \& Valdez Medina, J. L. (2013). Resiliencia: diferencias por edad en hombres y mujeres mexicanos. Acta de Investigación Psicológica, 3(1), 941-955. doi: 10.1016/S2007-4719(13)70944-X

Gooding, P. A., Hurst, A., Johnson, J., \& Tarrier, N. (2012). Psychological resilience in young and older adults. International Journal of Geriatric Psychiatry, 27, 22-270. doi: 10.1002 / gps. 2712

Granillo Velasco, A. D., Sánchez Aragón, R. \& Zepeda Goncen, G. D. (2020, julio). Bienestar Subjetivo: Medición integral y Validación en México. Revista Duanzary. En proceso de evaluación.

Grotberg, E. H. (2003). Resilience for Today: Gaining Strength from Adversity. Greenwood, SC: Praeger Publishers.

Hernández Sampieri, R., Fernández Collado, C., \& Baptista Lucio, P. (2014). Metodología de la investigación. México: McGraw-Hill.

Ito, T., Takenaka, K., Tomita, T., \& Agari, I. (2006). Comparison of ruminative responses with negative rumination as a vulnerability factor for depression. Psychological Reports, 99, 763 772. doi: 10.2466/PR0.99.7.763-772

Jiang, 1., Drolet, A., \& Kim, H. S. (2018). Age and support seeking: understanding the role of perceived social costs to others. Personality and Social Psychology Bulletin, 44(7), 11041116. doi: $10.1177 / 0146167218760798$

Johnson, D. P. \& Whisman, M. A. (2013). Gender differences in rumination: A meta-analysis. Personality and Individual Differences, 55(4), 367-374. doi: 10.1016 / j.paid.2013.03.019

Juárez Ramírez, C., Valdez Santiago, R. \& Hernández Rosete, D. (2005). La percepción de apyo social en mujeres con experiencia de violencia conyugal. Salud Mental, 28(4), 66-73.

Kaufman, S. B. (2016). The Differences between Happiness and Meaning in Life. Scientific American Blog Network. Descargado el 3 de Agosto, 2019 de: https://blogs.scientificamerican.com/beautiful-minds/the-differences-between-happinessand-meaning-in-life/

Kleiman, E. M. Chiara, A. M., Liu, R. T., Jager-Hyman, S. G., Choi, J. Y., \& Alloy, L. B. (2017). Optimism and well-being: a prospective multi-method and multidimensional examination of optimism as a resilience factor following the occurrence of stressful life events. Journal Cognition and Emotion, 31(2), 269-283. doi: 10.1080 / 02699931.2015.1108284 
Kompier, M. A. J., Taris, T. W., \& Veldhoven, M. V. (2012). Tossing and turning-insomnia in relation to occupational stress, rumination, fatigue and well-being. Scandinavian Journal of Work, Environment and Health, 38(3), 238-246. doi: 10.5271 / sjweh.3263

Londoño Pérez, C., Velasco Salamanca, M., Alejo Castañeda, I., Botero Soto, P., \& Vanegas, I. (2014). What makes us optimistic? Psychosocial factors as predictors of dispositional optimism in young people. Terapia Psicológica, 32(2), 153-164.

López Usero, S. (2016). Regulación emocional y género: un estudio exploratorio con estudiantado de grados feminizados. Tesis de grado. Descargada el 10 de agosto del 2019: http://repositori.uji.es/xmlui/handle/10234/161877

Matud, M. P., Ibañez, I., Bethencourt, J. M., Marrero, R., \& Carballeira, M. (2003). Structural gender differences in perceived social support. Personality and Individual Differences, 35(8), 1919-1929. doi: 10.1016/S0191-8869(03)00041-2

Michael, T., Halligan, S. L., Clark, D. M., \& Ehlers, A. (2007). Rumination in posttraumatic stress disorder. Depression \& Anxiety, 24(5), 307-317. doi: 10.1002 / da.20228

Morales Rodríguez, M. \& Díaz Barajas, D. (2011). Estudio comparativo de la resiliencia en adolescentes: el papel del género, la escolaridad y la procedencia. Revista de Psicología Uaricha, 8(17), 62-77.

Naci, H. \& Loannidis, J. P. A. (2015). Evaluation of Wellness Determinants and Interventions by Citizen Scientists. JAMA, 314(2), 121-122. doi: 10.1001 / jama.2015.6160

Nolen-Hoeksema, S. \& Aldao, A. (2011). Gender and age differences in emotion regulation and their relationship to depressive symptoms. Personality and Individual Differences, 51, 704708. doi: 10.1016/j.paid.2011.06.012

Nolen-Hoeksema, S., \& Davis, C. G. (2002). Positive responses to loss: Perceiving benefits and growth. In C. R. Snyder \& S. J. Lopez (Eds.), Handbook of positive psychology (pp. 598606). New York, NY, US: Oxford University Press.

Nolen-Hoeksema, S., Larson, J., \& Grayson, C. (1999). Explaining the gender difference in depressive symptoms. Journal of Personality and Social Psychology, 77(5), 1061-1072. doi: 10.1037/0022-3514.77.5.1061

Nolen-Hoeksema, S., McBride, A., \& Larson, J. (1997). Rumination and psychological distress among bereaved partners. Journal of Personality and Social Psychology, 72(4), 855-862. doi: 10.1037/0022-3514.72.4.855

Nolen-Hoeksema, S., Parker, L.E., \& Larson, J. (1994). Ruminative coping with depressed mood following loss. Journal of Personality and Social Psychology, 67, 92-104. doi: 10.1037/0022-3514.67.1.92

Organización Mundial de la Salud (2006). Constitución de la Organización Mundial de la Salud. Documentos Básicos: Suplemento 45ta. ed.. Recuperado el 10 de agosto de 2019 de: http://www.who.int/governance/eb/who_constitution_sp.pdf

Padrós Blázquez, F., Gutiérrez Hernández, C. Y. \& Medina Calvillo, M. A. (2015). Propiedades psicométricas de la Escala de Satisfacción con la vida (SWLS) de Diener en población de Michoacán (México). Avances de Psicología Latinoamericana, 33(2), 223-232. doi: 10.12804/apl33.02.2015.04

Palomar Lever, J. \& Gómez Valdez, N. E. (2010). Desarrollo de una escala de medición de la resiliencia con mexicanos (RESI-M). Interdisciplinaria, 27(1), 7-22. 
Peterson, C. (2000). The future of optimism. American Psychologist, 55(1), 44-55. doi: 10.1037/0003-066X.55.1.44

Pettus-Davis, C., Veeh, C. A., Davis, M., \& Tripodi, S. (2018). Gender differences in experiences of social support among men and women releasing from prison. Journal of Social and Personal Relationships, 35(9), 1161-1182. doi: 10.1177/0265407517705492

Prado Álvarez, R. \& del Águila Chávez, M. (2003). Diferencia en la resiliencia según género y nivel socioeconómico en adolescentes. Persona, 6, 179-196.

Procidano, M. E. (1992). The nature of perceived social support: Findings of meta-analytic studies.

En C. D. Spielberger \& J. N. Butler (Eds.), Advances in personality assessment (Vol. 9, pp. 126). Hilsdale, NJ:Erlbaum.

Puskar, K. R., Bernardo, L. M., Ren, D., Haley, T. M., Tark, K. G., Switala, J., \& Siemon, L. (2010). Self-esteem and optimism in rural youth: Gender differences. Contemporary Nurse, 34(2), 190-198. doi: 10.5172 / conu.2010.34.2.190

Reig, A. (2000). La calidad de vida en gerontología como constructo psicológico. Revista Española de Geriatría y Gerontología, 35(S2), 5-16.

Rood, L., Roelofs, J., Bogels, S.M., Nolen-Hoeksema, S., \& Schouten, E. (2009). The influence of emotion-focused rumination and distraction on depressive symptoms in non-clinical youth: A meta-analytic review. Clinical Psychology Review, 29, 607-616. doi: 10.1016 / j.cpr.2009.07.001

Sánchez Aragón, R. (2018). El Inicio y el Final de la Pareja: Variaciones en Admiración, Optimismo y Pasión Romántica. En R. Díaz Loving, L. I. Reyes Lagunes \& F. López Rosales (Eds.). La Psicología Social en México, Vol. XVII (pp. 999-1016). Monterrey: Asociación Mexicana de Psicología Social.

Sánchez Aragón, R. (en prensa). Apoyo de la pareja: Satisfacción, Capacidad para Recbirlo y Resiliencia en México. Revista Costarricense de Psicología.

Sansone, R. A. \& Sansone, L. A. (2012). Rumination: relationships with physical health. Innovations in Clinical Neuroscience, 9(2), 29-34.

Sarason, I. G., Levine, H. M., Basham, R. B., \& Sarason, B. R. (1983). Assessing social support: The Social Support Questionnaire. Journal of Personality and Social Psychology, 44(1), 127-139. doi: 10.1037/0022-3514.44.1.127

Schwaba, T., Robins, R. W., Priyanka, H. S., \& Bleidorn, W. (2019). Optimism development across adulthood and associations with positive and negative life events. Social Psychological and Personality Sciences, 1-10. doi: 10.1177/1948550619832023

Seligman, M. E. P. (1991). Learned optimism. New York: Knopf.

Seligman, M. E. P. (2002). Authentic happiness: Using the new positive psychology to realize your potential for lasting fulfillment. New York: Simon \& Schuster.

Seligman, M. E. P., Steen, T. A., Park, N., \& Peterson, C. (2005). Positive psychology progress: Empirical validation of interventions. American Psychologist, 60, 410 - 421. doi: 10.1037/0003-066X.60.5.410

Snyder, C. R. (1994). The psychology of hope: You can get here from here. New York: Free Press.

Sullivan, K. \& Davila, J. (2010). Introduction. En K. Sullivan \& J. Davila (Eds.). Support processes in intimate relationships (pp. xix-xxix). New York: Oxford University Press.

Sütterlin, S., Paap, M.C.S., Babic, S., Kübler, A. \& Vögele, C. (2012). Rumination and age: some things get better. Journal of Aging Research, 1-10. doi: 10.1155 / 2012/267327 
Tashiro, T., \& Frazier, P. (2003). "I'll never be in a relationship like that again": Personal growth following romantic relationship breakups. Personal Relationships, 10(1), 113-128. doi: 10.1111/1475-6811.00039

Verhofstadt, L. L. L., Lemmens, G. M. D., \& Buysse, A. (2013). Support-seeking, supportprovision and support-perception in distressed married couples: A multi-method analysis. Journal of Family Therapy, 35(3), 320-339. doi: 10.1111/1467-6427.12001

Watkins, E.R., Mullan, E., Wingrove, J., Rimes, K., Steiner, H., Bathurst, N., Eastman, R., \& Scott, J. (2011). Rumination-focused cognitive-behavioral therapy for residual depression: phase II randomized controlled trial. British Journal of Psychiatry, 199(4), 317-322. doi: 10.1192 / bjp.bp. 110.090282

Webber, K. C. \& Smokowski, P. R. (2018). Assessment of adolescent optimism: measurement invariance across gender and race/ethnicity. Journal of Adolescence, 68, 78-86. doi: 10.1016 / j.adolescence.2018.06.014

Zepeda Goncen, G. D. \& Sánchez Aragón, R. (2019). Efectos del Apego, Afecto y Capacidad de Recibir Apoyo en la Salud de la Pareja. Revista Psicologìa e Eduçao (On-Line), 2(1), 66-76.

Agradecimientos: A la Dirección General de Apoyo al Personal Académico de la Universidad Nacional Autónoma de México por financiar el proyecto: PAPIIT IN304919 "Factores protectores y de riesgo a la salud en parejas sanas y con enfermedad crónico-degenerativa"

Contribución de los autores: a) Concepción y diseño del trabajo; b) Adquisición de datos; c) Análisis e interpretación de datos; d) Redacción del manuscrito; e) revisión crítica del manuscrito.

R. S. A. ha contribuido en a,b,c,d,e.

Editora científica responsable: Dra. Cecilia Cracco 